\title{
miR-16-2* Interferes with WNT5A to Regulate Osteogenesis of Mesenchymal Stem Cells
}

\author{
Lijun Duan $^{\mathrm{a}, \mathrm{b}} \quad \mathrm{He}$ Zhao $^{\mathrm{a}} \quad$ Yang Xiong $^{\mathrm{a}} \quad$ Xiangsheng Tang ${ }^{\mathrm{c}} \quad$ Yongdong Yang $^{\mathrm{a}}$ \\ Zhenguo $\mathrm{Hu}^{\mathrm{a}}$ Chuanhong Lia Sixue Chen ${ }^{\mathrm{a}}$ Xing Yu \\ aDepartment of Orthopedics, Dongzhimen Hospital, Beijing University of Chinese Medicine, Beijing, \\ bDepartment of Orthopedics, Bayannaoer City Hospital, Bayannaoer City, 'Department of Orthopedics, \\ China-Japan Friendship Hospital,Beijing University of Chinese Medicine, Beijing, China
}

\section{Key Words}

Mesenchymal stem cells $\bullet$ miR-16-2* $\bullet$ Osteoporosis $\bullet$ Osteogenesis $\bullet$ Bone formation $\bullet$ WNT5A

\begin{abstract}
Background/Aims: Osteoporosis is a bone metabolic disease characterized by a systemic impairment of bone mass, which results in increased propensity of fragility fractures. A reduction in the differentiation of MSCs into osteoblasts contributes to the impaired bone formation observed in osteoporosis. Mesenchymal stem cells (MSCs) are induced to differentiate into preosteoblasts, which are regulated by the signaling cascades initiated by the various signals, including miRNAs. miR-16-2* is a newly discovered miRNA that participates in diagnosis and prognosis of hepatocellular carcinoma, cervical cancer and chronic lymphocytic leukemia. However, the effect of miR-16-2* on the regulation of osteoblast differentiation and the mechanism responsible are still unclear. Here we discuss the contribution of miR-16$2^{*}$ to osteoporosis, osteoblast differentiation and mineralization. Methods: The expression pattern of miR-16-2* during osteogenesis or in osteoporosis bone samples was validated by quantitative real-time PCR (qRT-PCR). The human bone marrow mesenchymal stem cells (hBMSCs) were induced to differentiate into osteoblasts by osteogenic induced medium containing dexamethasone, ascorbate-2-phosphat, beta-glycerophosphate and vitamin-D3. The target genes of miR-16-2* were predicted by TargetScan and PicTar. The mRNA and protein levels of osteogenic key markers were detected using qRT-PCR or western blot respectively. The WNT signal activity was analyzed by TOP/FOP reporter assay. Results: The expression of miR-16-2* in patient bone tissue with osteoporosis was negatively correlated with bone formation related genes. During osteoblast differentiation process, the expression of miR-16$2^{*}$ was significantly decreased. Upregulation of miR-16-2* in hBMSCs impaired the osteogenic differentiation while the downregulation of miR-16-2* increased this process. Upregulation the expression of miR-16-2* could also block the WNT signal pathway by directly target

L. Duan and $\mathrm{H}$. Zhao contributed equally to this work.

\begin{tabular}{ll}
\hline Xing Yu & Department of Orthopedics, Dongzhimen Hospital, Beijing University of Chinese Medicine \\
& NO.5 Haiyuncang Street, Dong cheng District, Beijing100700 (China) \\
& Tel. 010-84013324, Fax 010-84013324, E-Mail yuxingbucm@sina.com
\end{tabular}
\end{abstract}


WNT5A. Furthermore, knockdown of miR-16-2* could promote the activation of RUNX2, possibly by lifting the inhibitory effect of miR-16-2* on WNT pathway. Conclusion: Taken together, we report a novel biological role of miR-16-2* in osteogenesis through regulating WNT5A response for the first time. Our data support the potential utilization of miRNA-based therapies in regenerative medicine.

\section{Introduction}

In countries with large aging populations, such as China [1], osteoporosis is a common and systemic bone metabolic disease characterized by loss of bone mass and increase in fragility fractures. Osteogenesis is a complex multi-step process involving the differentiation of mesenchymal stem cells (MSCs) into osteoblast progenitor cells, preosteoblasts, osteoblasts and osteocytes. By secreting bone matrix protein alkaline phosphatase (ALP) and that induce bone matrix mineralization, osteoblasts have been widely accepted as the major cells that contribute to osteogenesis and bone formation [2, 3]. A reduction in the differentiation of MSCs into osteoblasts contributes to the impaired bone formation observed in osteoporosis. Therefore, understanding the underlying mechanism involved in the osteoblast differentiation from MSCs and activation processes is in urgent need for the treatment of osteogenesis imbalanced diseases. Recent research has found MSCs are induced to differentiate into preosteoblasts, which are tightly regulated by several factors, including microRNAs (miRNAs).

miRNAs are a class of evolutionarily conserved, small noncoding RNA molecules (18-25 nucleotides), which control gene expression at a posttranscriptional level through binding to the seed sequences of the 3'-untranslated region (UTR) of target messenger RNAs (mRNAs) and mediating the degradation of mRNA or translation inhibition [4, 5]. miRNAs have been shown to play critical roles in a wide variety of biological processes, including proliferation, lineage commitment, differentiation, cell cycle, invasion and apoptosis [6-10]. Recently, increasing evidences have indicated that bone development and homeostasis is regulated by miRNAs [11-14].

miR-15/16 family [15-18] encompasses the miR-15a/16-1 cluster (on chromosome 13q14,) the miR-15b/16-2 cluster (on chromosome 3q25), and the miR-195/497 cluster (on chromosome 17p13). miR-16-2 on chromosome 3 rather than of miR-16-1 on chromosome 13. Whereas miR-15a is highly similar to miR-15b, miR-16-1 is identical to miR-16-2. Thus, it could be speculated that both clusters control a similar set of target genes and may have overlapping functions. However, the biological role of miR-15b/16-2 is still controversial. The central role of the miR-15a/16-1 cluster in oncogenesis has been extensively demonstrated. The circulatory miR-16-2 level has been established as critical factor for diagnosing hepatocellular carcinoma, cervical cancer and chronic lymphocytic leukemia [19-21]. However, the central role of this miRNA in osteogenesis is still far from being elucidated.

Recently, we found that mature miR-16-2* (also name as miR-16-2-3p) levels are abnormally expressed in bone samples of patients with osteoporosis, which suggest an important role of miR-16-2* in the regulation of MSC osteogenic differentiation. The aim of this study is to characterize the expression of miR-16-2* in osteoporosis and investigate its effects on osteoblast differentiation, as well as the potential molecular mechanisms.

\section{Materials and Methods}

Clinical bone specimen preparation and RNA extraction

Forty-six clinical bone specimens were collected from the Dongzhimen Hospital affiliated to Beijing University of Chinese Medicine. The clinical bone sample was originally from patients at the age between 55 and 70 years old. Exclusive criteria: individuals with diabetes, malignancy or other severe diseases 


\section{Cellular Physiology Cell Physiol Biochem 2018;51:1087-1102 and Biochemistry \begin{tabular}{l|l} 
DOI: 10.1159/000495489 & (c) 2018 The Author(s). Published by S. Karger AG, Basel \\
www.karger.com/cpb
\end{tabular}

in the previous 5 years; inclusive criteria: patients who with low-energy fractures [22, 23]. All of the clinical procedures were approved by the IRB of Dongzhimen Hospital affiliated to Beijing University of Chinese Medicine.

\section{Osteogenic differentiation \\ Osteogenic induction was}

Table 1. The primers used in this study

\begin{tabular}{lcc}
\hline Gene name & forward primer & reverse primer \\
\hline GAPDH & 5'-GGAGCGAGATCCCTCCAAAAT-3' & 5'-GGCTGTTGTCATACTTCTCATGG-3' \\
RUNX2 & 5'-CTTCACAAATCCTCCCCAAG-3' & 5'-GAATGCGCCCTAAATCACTG-3' \\
ALP & 5'-GCGCAAGAGACACTG AAATAT-3' & 5'-TGGTGGAGCTGACCCTTGAG-3' \\
OSX & 5'-CACCAGGTCCAGGCAACA-3' & 5'-GAGCAAAGTCAGATGGGTAAGT-3' \\
OCN & 5'-GAAGCCCAGCGGTGCA-3' & 5'-CACTACCTCGCTGCCCTCC-3' \\
COL1A & 5'-GAGGGCCAAGACGAAGACATC-3' & 5'-CAGATCACGTCATCGCACAAC-3' \\
OPN & 5'-CTCCATTGACTCGAACGACTC-3' & 5'-CAGGTCTGCGAAACTTCTTAGAT-3' \\
WNT5A & 5'-CAAATAGGCAGCCGAGAGAC-3' & 5'-CTCTAGCGTCCACGAACTCC-3' \\
CTNNB1 & 5'-AAAGCGGCTGTTAGTCACTGG-3' & 5'-CGAGTCATTGCATACTGTCCAT-3' \\
LRP5 & 5'-AATCAACAAGCCACCCTCTG-3' & 5'-GGCTCCACCAACATACTCGT-3' \\
LRP6 & 5'-ACCCTGCAGCACTGATGTCT-3' & 5'-GTTCTCCTCCGCTGACAAGT-3' \\
\hline
\end{tabular}

performed as described previously [23]. Cells were grown in 6-well plates, when reached $70-80 \%$ confluence, cells were transfected. Twenty-four hours after transfection, osteogenic differentiation was started on hBMSCs by replacing the growth medium with an osteogenic differentiation medium consisting of Dulbecco's modified Eagle's medium (DMEM), 10\% FCS (SigmaAldrich, Munich, Germany), $4 \mathrm{mM}$ Lglutamine, $10 \mathrm{nM}$ dexamethasone (Sigma-Aldrich), $10 \mathrm{nM}$ calcitriol (1 $\alpha, 25$-dihydroxyvitamin D3; Sigma), $150 \mathrm{mM}$ ascorbate-2-phosphat, $10 \mathrm{mM}$ beta-glycerophosphate (Sigma-Aldrich). Medium was refreshed every two days. Measurement of alkaline phosphatase (ALP) expression and ALPase activity served as early marker for osteogenesis and were detected by ALP staining and quantitative ALP activity measurement. Calcium deposits as late marker for osteogenesis were confirmed by Alizarin Red S staining.

ALP staining and ALPase activity measurement

ALP staining was monitored using an ALP staining kit (Beyotime® Institute of Biotechnology, Shanghai, China) according to the manufacturer's protocol. Cellular ALP activity was measured with an ALP activity kit (GENMED, Shanghai, China). Briefly, the cells were washed twice with PBS, and incubated in an ALP staining solution (Sigma-Aldrich) at $37^{\circ} \mathrm{C}$ for $20 \mathrm{~min}$ with gentle shaking in the dark. ALPase activity was measured with a spectrophotometric method by a p-Nitrophenyl Phosphate Liquid Substrate System (Sigma-Aldrich). The absorbance at $405 \mathrm{~nm}$ of each well was assayed with a microplate reader (BD Biosciences, Franklin Lakes, NJ, USA) according to the manufacturer's instruction. The ALPase activity was normalized to the protein concentration.

\section{Alizarin red staining (ARS)}

Cells were washed with PBS, fixed in 4\% paraformaldehyde (Sigma-Aldrich) for $10 \mathrm{~min}$ at room temperature. After removing the fixative, cells were rinsed with double-distilled $\mathrm{H}_{2} \mathrm{O}$ and stained with 40 $\mathrm{mM}$ alizarin red (Sigma), with $\mathrm{pH} 4.2$ for $30 \mathrm{~min}$ with gentle agitation at room temperature. Cells were then rinsed with double-distilled $\mathrm{H}_{2} \mathrm{O}$ to remove the excess dye and subsequently photographed. For quantification, to eluted the bound dye, stained cells were incubated in $800 \mu \mathrm{L}$ of acetic acid for 30 min at room temperature as previously described [24], and then measured using a microplate reader (BD Biosciences, Franklin Lakes, NJ, USA) at $405 \mathrm{~nm}$.

\section{Quantitative real-time PCR ( $q$ RT-PCR) analysis}

Total RNA was extracted using Trizol reagent (Gibco, Carlsbad, CA, USA) according to the manufacturer's instructions, and was reverse transcribed into cDNA using the PrimeScript RT Reagent Kit (\#DRR037A; TaKaRa). The complementary DNA was synthesized from $1 \mu \mathrm{g}$ total RNA using the PrimeScript RT Reagent Kit (\#DRR037A; TaKaRa). The amplification of marker genes was performed by quantitative real-time PCR (qRT-PCR). qRT-PCR was performed with an SYBR Green qPCR kit (Toyobo, Osaka, Japan) on an ABI PRISM 7900 Real-time PCR System (Applied Biosystems, Carlsbad, CA, USA). The primers used in this study are shown in Table 1. The extraction, reverse transcription, and qPCR of the miRNA were realized by use of the corresponding kit from GeneCopoeia. The primers for miR-16-2* and the endogenous control U6 were purchased from GeneCopoeia. 


\section{Cellular Physiology Cell Physiol Biochem 2018;51:1087-1102 and Biochemistry \begin{tabular}{l|l} 
DOI: 10.1159/000495489 & (c) 2018 The Author(s). Published by S. Karger AG, Basel \\
www.karger.com/cpb
\end{tabular} \\ Duan et al.: miR-16-2* Regulates Osteogenesis Via WNT Pathway}

\section{MiRNA and siRNA transfection}

Transfection was performed using Lipofectamine 3000 (Invitrogen, Carlsbad, CA, USA), according to the manufacturer's instructions. The miRNA mimic, inhibitor, and their negative controls for miR-16-2*; small interfering RNA (siRNA) duplex oligos targeting WNT5A mRNA and miR-16-2* mRNA; and negative duplex oligos (siRNA negative controls) were purchased from Genepharma (Shanghai, China). Cells were cultured overnight in six-well or twenty-four plates until they reached $50 \%$ confluence were transfected with miRNA mimics/inhibitors/siRNA or negative control at a final concentration of $100 \mathrm{nM}$ according to the manufacturer's instructions of Lipofectamine 2000.

\section{TOP/FOP reporter assay}

The activity of the Wnt signaling pathway was detected by a Wnt signal reporter assay using the TOPglow/FOPglow TCF reporter kit (Millipore). TOPglow (cat. \# 21-204) is transfection grade T cell factor (TCF) reporter plasmid which containing four copies of the TCF binding site (WT) upstream of E1B TATA box and luciferase open reading frame. FOPglow (cat. \# 21-205) is transfection grade T cell factor (TCF) reporter plasmid containing three copies of the TCF binding site (mutated) upstream of E1B TATA box and luciferase open reading frame. This plasmid serves as a negative control to TOPglow. Cells were seeded in 6-well plates and transfected with TOPglow and FOPglow according to the manufacturer's instructions. All transfections were done in triplicate and repeated at least 3 times.

\section{Western blotting analysis}

Cells were lysed in RIPA buffer (Beyotime, Shanghai, China) supplemented with roteinase and phosphatase inhibitors (Roche). Protein was manually scraped from culture plates and then sonicated and quantified using the BCA Protein Assay Kit (Beyotime). Protein fractions were separated using 10\% SDS/ PAGE and transferred to a PVDF membrane (UltraCruz). Then, the membranes were blocked in 5\% BSA for 1 hour, incubated with specific antibodies at $4{ }^{\circ} \mathrm{C}$ overnight, and then with secondary antibodies for 1 hour. The blots were detected with ECL reagent (Millipore).

\section{Luciferase reporter assay}

TargetScan (http://www.targetscan.org) and PicTar (http://pictar.mdc-berlin.de/) software were used in this study to identify miR-16-2* potential targets. An 80- to 100-bp synthetic fragment of the 3'UTR of SMAD5, BMP4, BMPR1A, WNT5A, FZD3, FZD5 containing the putative miR-16-2* recognition sequence and corresponding mutated 3'UTR were subcloned into the psiCHECK-2 vector (Promega, Southampton, UK) downstream of the renilla luciferase reporter gene. HEK293T cells were seeded in 24-well plates at a density of $1 \times 10^{5}$ cells per well. Overnight HEK293T cells were co-transfected with the luciferase reporter vector and miR-16-2* mimic, miRNA mimic negative control (miR-NC), miR-16-2* inhibitor (miR-16-2*I) or miRNA inhibitor negative control (miR-NCI). Transfection assay was conducted using lipofectamine 3000 (Invitrogen). Firefly and Renilla luciferase activities were detected $48 \mathrm{~h}$ after transfection using the dual-luciferase reporter assay system (Promega Corporation, Madison, WI, USA). The Renilla values were normalized to firefly luciferase. Each experiment was repeated in triplicate.

\section{Statistical analysis}

Data are presented as the mean \pm SD from at least three time independent experiments. Significant differences between groups or multiple groups were analyzed using Student's $t$-tests or one-way ANOVA respectively. Spearman rank order was used to analyze the correlations between variables. Differences were considered statistically significant at ${ }^{*} \mathrm{P}<0.05,{ }^{* *} \mathrm{P}<0.01$ and ${ }^{* * *} \mathrm{P}<0.001$.

\section{Results}

miR-16-2* is negative correlated with bone formation and downregulated during osteogenic differentiation

To determine whether miR-16-2* is related to bone formation, the expression levels of miR-16-2* in clinical bone specimen were analyzed using qRT-PCR. Results showed that the expression of miR-16-2* in clinical bone specimen was negative correlated with the 


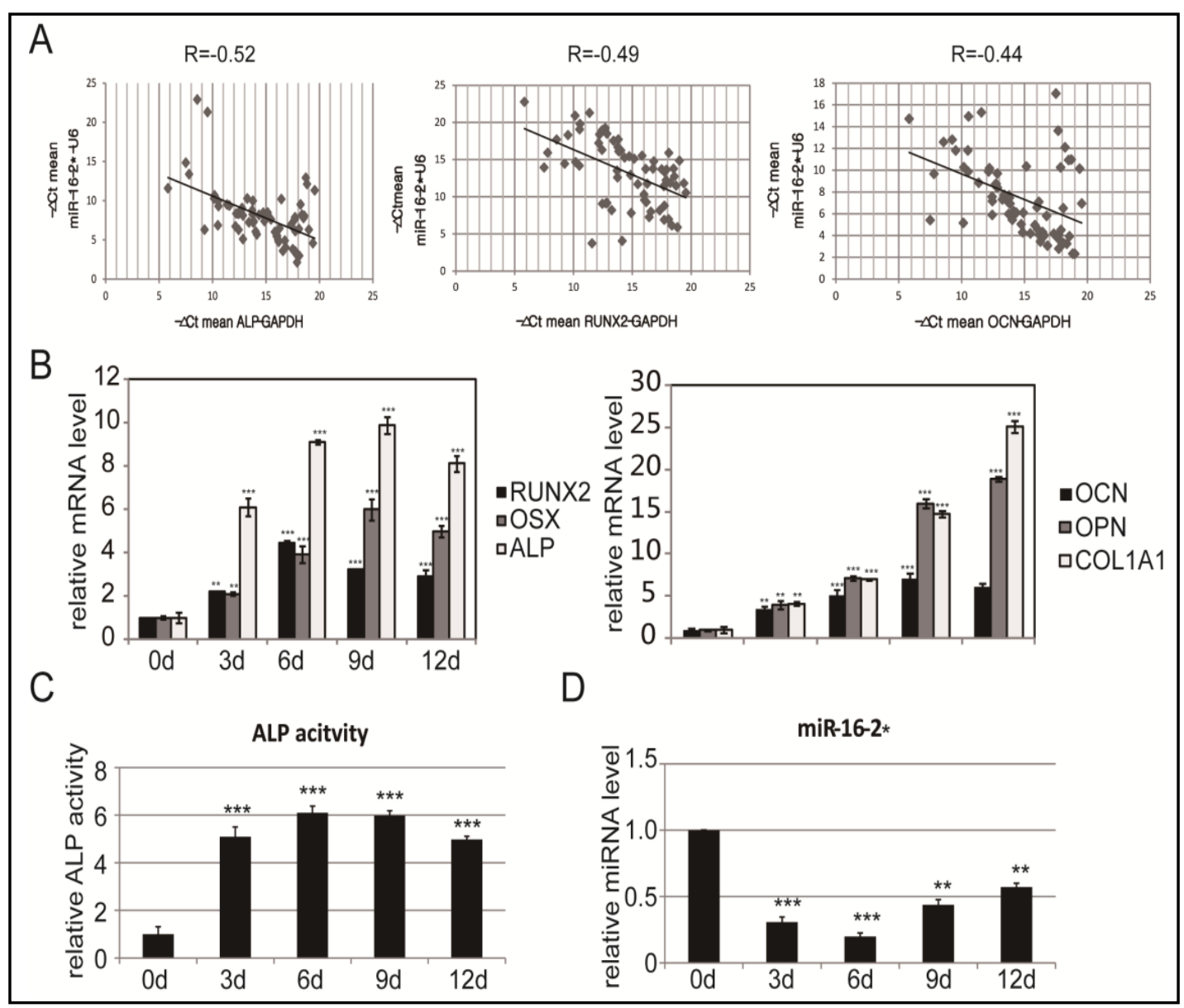

Fig. 1. The correlation and expression pattern of miR-16-2*. (A) The correlation between the expression of osteogenic related genes and miR-16-2* expression in clinical osteoporosis samples. (B) qRT-PCR was used to analyze the expression of key osteoblast markers. (C) The activity of ALPase was detected on day 6 of osteoblast differentiation. (D) The dynamic expression of miR-16-2* during osteoblast differentiation of hBMSCs. All data were presented as the mean \pm SD from three independent experiments, and U6 was used as an internal normalization control.

expression of key genes of bone formation (Fig. 1A), indicating that miR-16-2* was involved in the progression of bone formation. We then detected the expression levels of miR-16-2* during osteogenic differentiation of hBMSCs. A number of key osteoblast markers, including RUNX2, OSX, OCN, ALP, OPN and COL1A1, were used as phenotypic markers of osteogenic differentiation. The expressions of all the markers above and the activity of ALPase were obviously increased in the osteogenic medium treated cells, indicating that osteogenic differentiation had been successfully induced (Fig. 1B and 1C). Results of miRNA specific qRT-PCR showed that miR-16-2* expression levels decrease significantly in along with the time course during osteoblast differentiation (Fig. 1D). These results demonstrated that miR-16-2* may have a role in the progression of osteogenic differentiation.

\section{Forced expression of miR-16-2* in BMSCs suppressed osteoblast differentiation}

In order to assess the physiological role of miR-16-2* on osteogenic differentiation and bone formation, BMSCs were transfected with either miR-16-2* mimic or control and cultured under osteogenic induced medium. MiRNA specific qRT-PCR analysis confirmed that transfection of BMSCs with control does not affect endogenous miR-16-2* level, while miR-16-2* mimic transfection markedly increased miR-16-2* level (Fig. 2A) and resulted in significantly reduced levels of key transcription factors for osteogenic differentiation, 
RUNX2, OSX and ALP, as well as known osteoblast differentiation and lineage commitment marker genes, OCN, OPN and COL1A1 (Fig. 2B). Similar changes were also observed in western blotting analysis of these genes (Fig. 2C). miR-16-2* mimic transfection weaken ALP staining, decreased ALPase activity in differentiated cells and markedly suppressed calcification of induced cells which detected by ARS staining (Fig. 2D-F). Collectively, these results demonstrated that forced expression of miR-16-2* can suppress the progression of osteoblast differentiation.

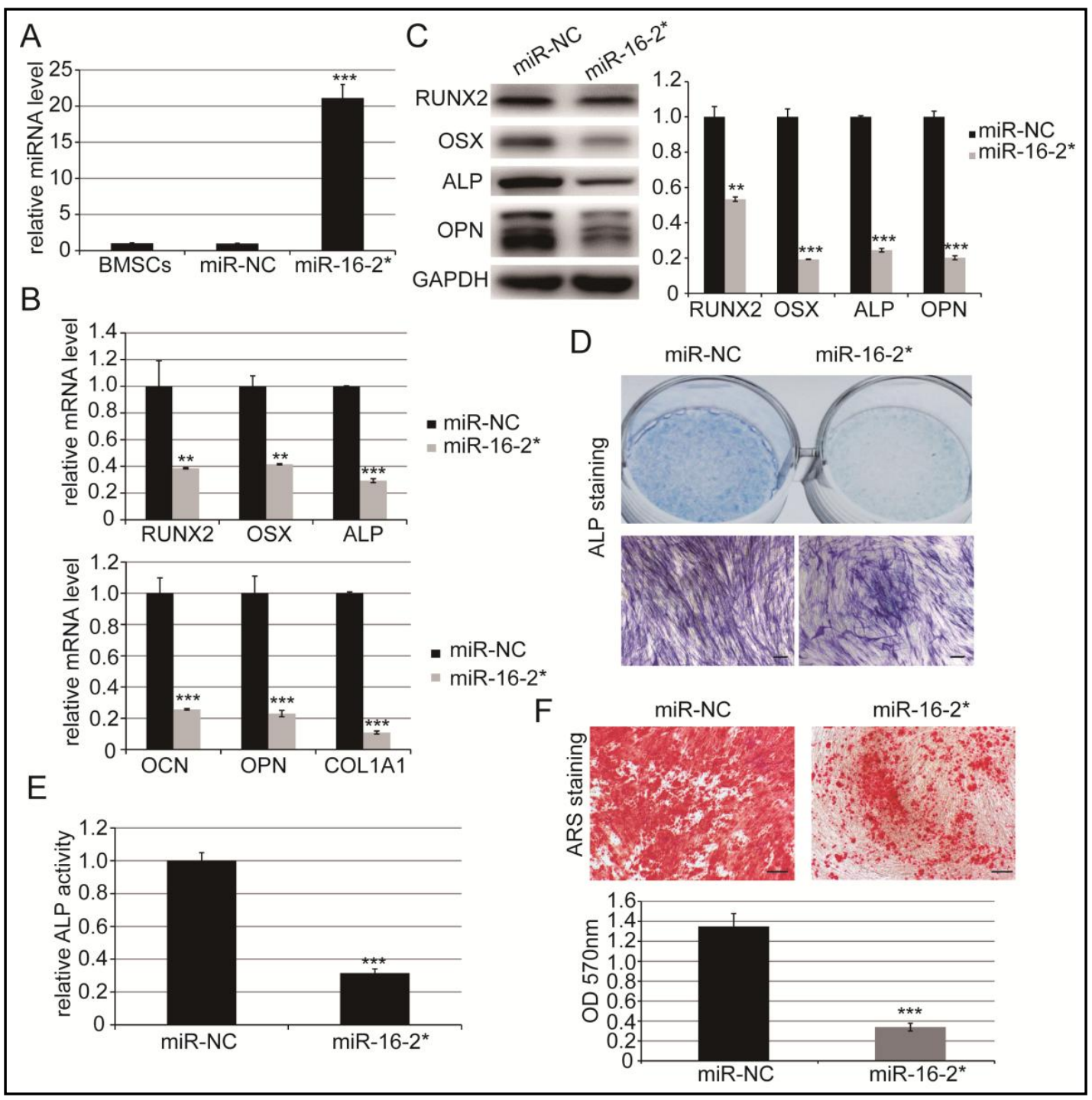

Fig. 2. Forced expression of miR-16-2* in BMSCs suppressed osteoblast differentiation. (A) MiRNA specific qRT-PCR was used to detect the expression of miR-16-2*. (B) qRT-PCR was used to analyze the expression of key transcription factors and osteoblast markers on day 6 of induced cells. (C) Western blotting analysis was used to analyze the protein level of key osteoblast markers on day 6 of induced cells. (D and E) ALP staining and ALPase activity assay were performed to indicate ALP expression and ALPase activity on day 6 of induced cells. (F) ARS was performed to indicate mineral deposition on day 12 of induced cells. GAPDH was used as an internal control in RT-PCR and western blot analysis of genes. U6 was used as an internal normalization control of miRNA. Quantitative data were presented as the mean \pm SD ( $n=3$ ), ${ }^{*} \mathrm{P}<0.05$; ${ }^{* *} \mathrm{P}<0.01 ;{ }^{* * *} \mathrm{P}<0.001$ compared with their negative control. All the staining and western blot images were confirmed by at least three repeated trails. Scale bar is $100 \mathrm{~mm}$. 


\section{Downregulation of miR-16-2* enhances the osteoblast differentiation}

In order to further explore the effects of miR-16-2* for osteoblast differentiation, we suppressed the expression of miR-16-2* in hBMSCs with inhibitors (Fig. 3A). Results showed that downregulation of miR-16-2* markedly increased the mRNA expression levels of key transcription factors for osteoblast differentiation, RUNX2, OSX and ALP, as well as known osteoblast differentiation and lineage commitment marker genes, OCN, OPN and COL1A1 (Fig. 3B). Similar changes were also observed at protein level of these genes through western

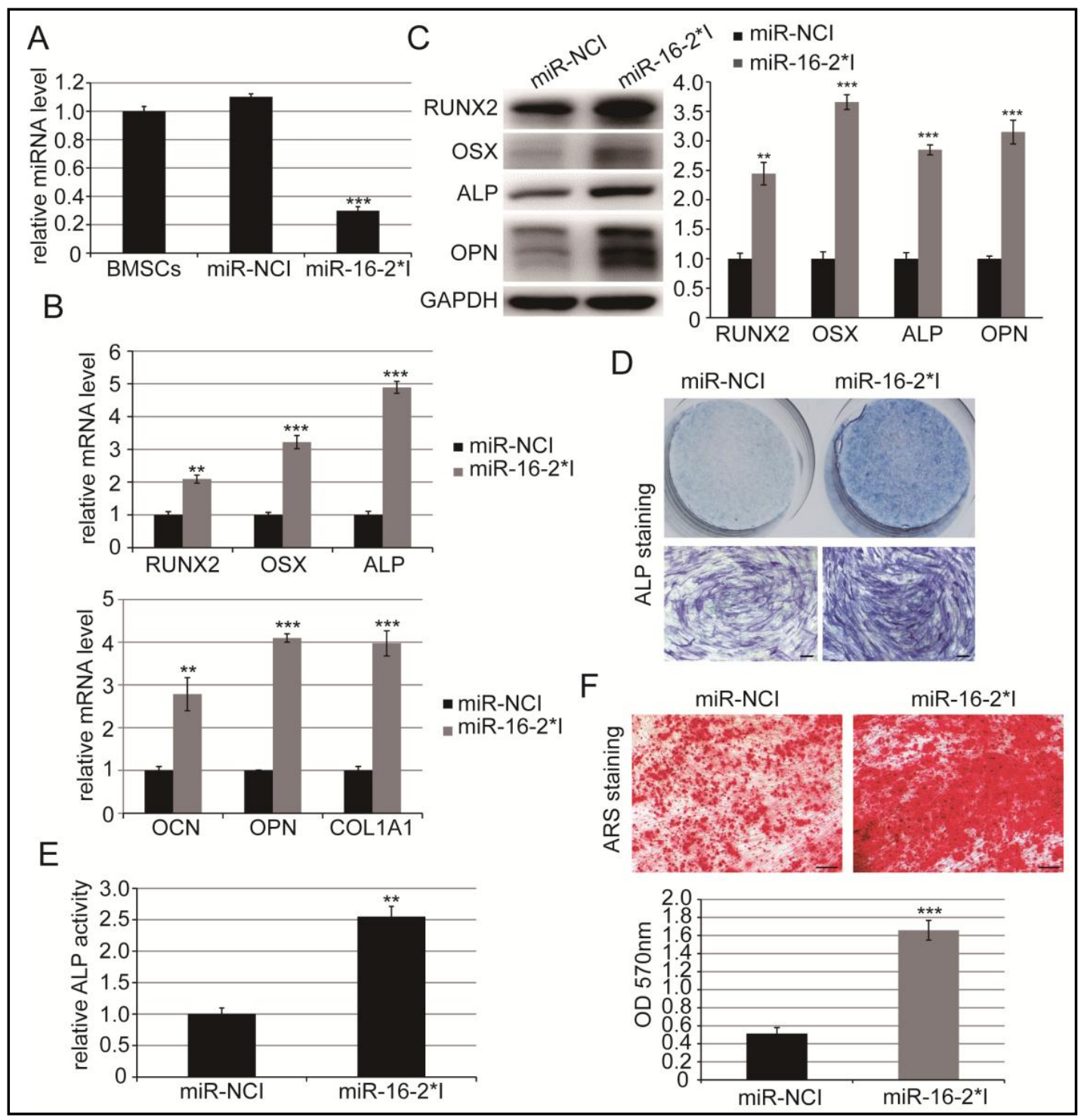

Fig. 3. Downregulation of miR-16-2* enhances the osteoblast differentiation. (A) MiRNA specific qRT-PCR was used to detect the expression of miR-16-2* in hBMSCs with miR-16-2* inhibitor. (B) qRT-PCR was used to analyze the expression of key transcription factors and osteoblast markers on day 6 of induced cells. (C) Western blotting analysis was used to analyze the protein level of key osteoblast markers on day 6 of induced cells. (D and E) ALP staining and ALPase activity assay were performed to indicate ALP expression and ALPase activity on day 6 of induced cells. (F) ARS staining was performed to indicate mineral deposition on day 12 of induced cells. GAPDH was used as an internal control in RT-PCR and western blot analysis of genes. U6 was used as an internal normalization control of miRNA. Quantitative data were presented as the mean $\pm \mathrm{SD}(\mathrm{n}=3),{ }^{*} \mathrm{P}<0.05 ;{ }^{* *} \mathrm{P}<0.01 ;{ }^{* *} \mathrm{P}<0.001$ compared with their negative control. All the staining and western blot images were confirmed by at least three repeated trails. Scale bar is $100 \mathrm{~mm}$. 


\section{Cellular Physiology Cell Physiol Biochem 2018;51:1087-1102 \begin{tabular}{ll|l} 
and Biochemistry Published onIIne: 24 November 2018 & $\begin{array}{l}\text { (c) } 2018 \text { The Author(s). Published by S. Karger AG, Basel } \\
\text { www.karger.com/cpb }\end{array}$ \\
\hline
\end{tabular}

blotting analysis (Fig. 3C). The ALP staining and activity of ALPase in the miR-16-2* inhibitor (miR16-2*I) transfected cells were significantly increased, compared with the miRNA inhibitor negative control (miR-NCI) transfected cells (Fig. 3D, E). ARS staining to indicate calcification was also enhanced markedly in the miR16-2*I transfected cells (Fig. 3F). These data further confirmed that downregulation of miR-16-2* can enhance the osteoblast differentiation of BMSCs.

WNT5A is a direct target gene of miR-16-2*

To identify targets mediating the inhibitory effect of miR-16-2* on osteoblast differentiation, we predicted the potential targets in silico using various databases. Take complementarity of the miRNA seed sequence into account, TargetScan and PicTar showed that SMAD5, BMP4, BMPR1A, WNT5A, FZD3, FZD5, have binding site of miR-16-2* seed sequence in their 3'UTR (Table 2). To further confirm the interaction between miR-16-2* seed sequence and the $3^{\prime} U T R$ of its potential target genes, luciferase reporter assay was performed (Fig. 4A and 4B). Results showed that miR-16-2* directly bound on the 3'UTR of WNT5A mRNA, while not targeted the 3'UTR of WNT5A mRNA with mutant seed region or other genes 3'UTR as mentioned above (Fig. 4C). We then analyzed the regulation effect of miR-16-2* on the expression of its potential target WNT5A. Consistent with the luciferase reporter assays, qRT-PCR and western blotting analyses also showed that miR-16-2* mimic decreased WNT5A expression at protein level most significantly (Fig. 4D). These data suggested that miR-16-2* can bind the 3'UTR of WNT5A mRNA and regulate its expression at the posttranscriptional level in hBMSCs.

Downregulation of WNT5A simulated the inhibitory effect of miR-16-2* on osteoblast differentiation

To further confirm the interaction between miR-16-2* and WNT5A, we suppressed the endogenous expression level of WNT5A by siRNA. qRT-PCR and western blotting confirmed that WNT5A was significantly suppressed by siWNT5A at both mRNA and protein levels (Fig. $5 A, B)$. Then we analyzed the effect of WNT5A downregulation on osteoblast differentiation of hBMSCs. qRT-PCR results showed that transfection of siWNT5A sharply decreased the mRNA expression levels of RUNX2, OSX and ALP, as well as known osteoblast differentiation and lineage commitment marker genes, OCN, OPN and COL1A1, compared with the transfection of siRNA negative control (siNC) (Fig. 5C). Similar changes were also observed at protein levels of these genes through western blotting analysis (Fig. 5D). The ALP staining and the activity of ALP were decreased significantly in the siWNT5A-transfected cells, compared with the siNC transfected cells (Fig. 5E, F). Calcium deposition in the siWNT5A transfected cells was sharply less than the siNC transfected cells, indicated by ARS staining (Fig. 5G). These results revealed that repression of target gene WNT5A can simulate the effect of miR16-2* overexpression on osteoblast differentiation of hBMSCs, which confirmed that the interaction between miR-16-2* and WNT5A plays a pivotal role of in regulation of osteoblast differentiation.

miR-16-2* interferes with WNT signaling to regulate osteoblast differentiation

WNT5A is an important member of the WNT pathway. To further explore the interaction between miR-16-2* and WNT signaling, we performed a dosage experiment to block WNT signaling by DKK1. WNT signaling inhibitor, DKK1 of different concentrations was added into the osteoblast induction medium during osteogenic differentiation of hBMSCs. Results 


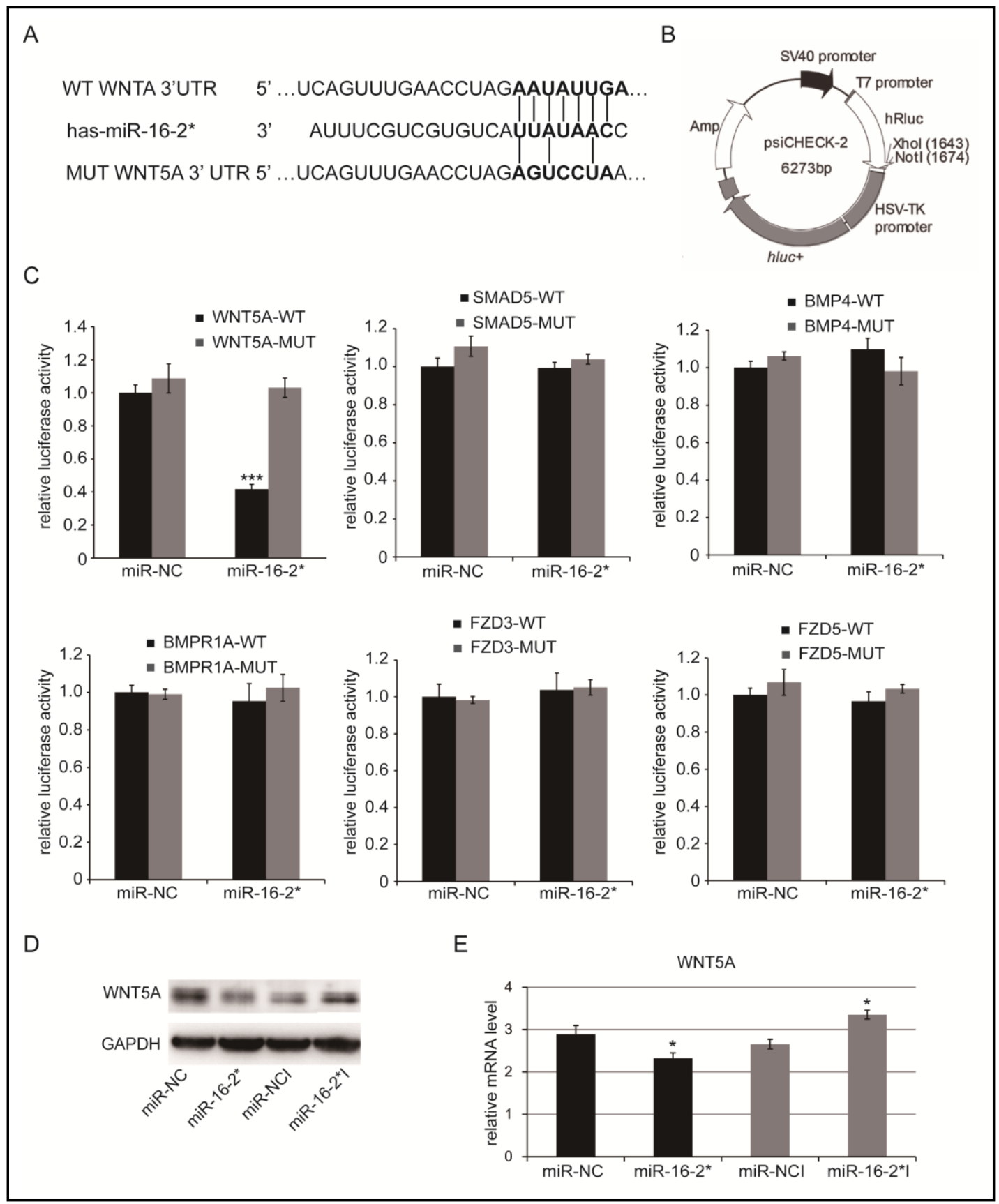

Fig. 4. miR-16-2* direct targets the $3^{\prime}$ UTR of WNT5A and regulates its expression. (A and B) The binding seed sequence was predicted by TargetScan and PicTar. (B) The wild-type (WT) or mutant-type (MUT) constructs were inserted into the psiCHECK-2 reporter vector. $(C)$ Luciferase reporter assay was performed to confirm the matching of miR-16-2* and the 3'UTR of WNT5A, SMAD5, BMP4, BMPR1A, FZD3 and FZD5. (D) Western blotting and qRT-PCR analyzed the effect of miR-16-2* on WNT5A expression. GAPDH was used as an internal control in RT-PCR and western blot analysis of genes. U6 was used as an internal normalization control of miRNA. Quantitative data were presented as the mean $\pm \mathrm{SD}(\mathrm{n}=3),{ }^{*} \mathrm{P}<0.05 ;{ }^{* *} \mathrm{P}<0.01$; $^{* * *} \mathrm{P}<0.001$ compared with their negative control. All the staining and western blot images were confirmed by at least three repeated trails. Scale bar is $100 \mathrm{~mm}$. 


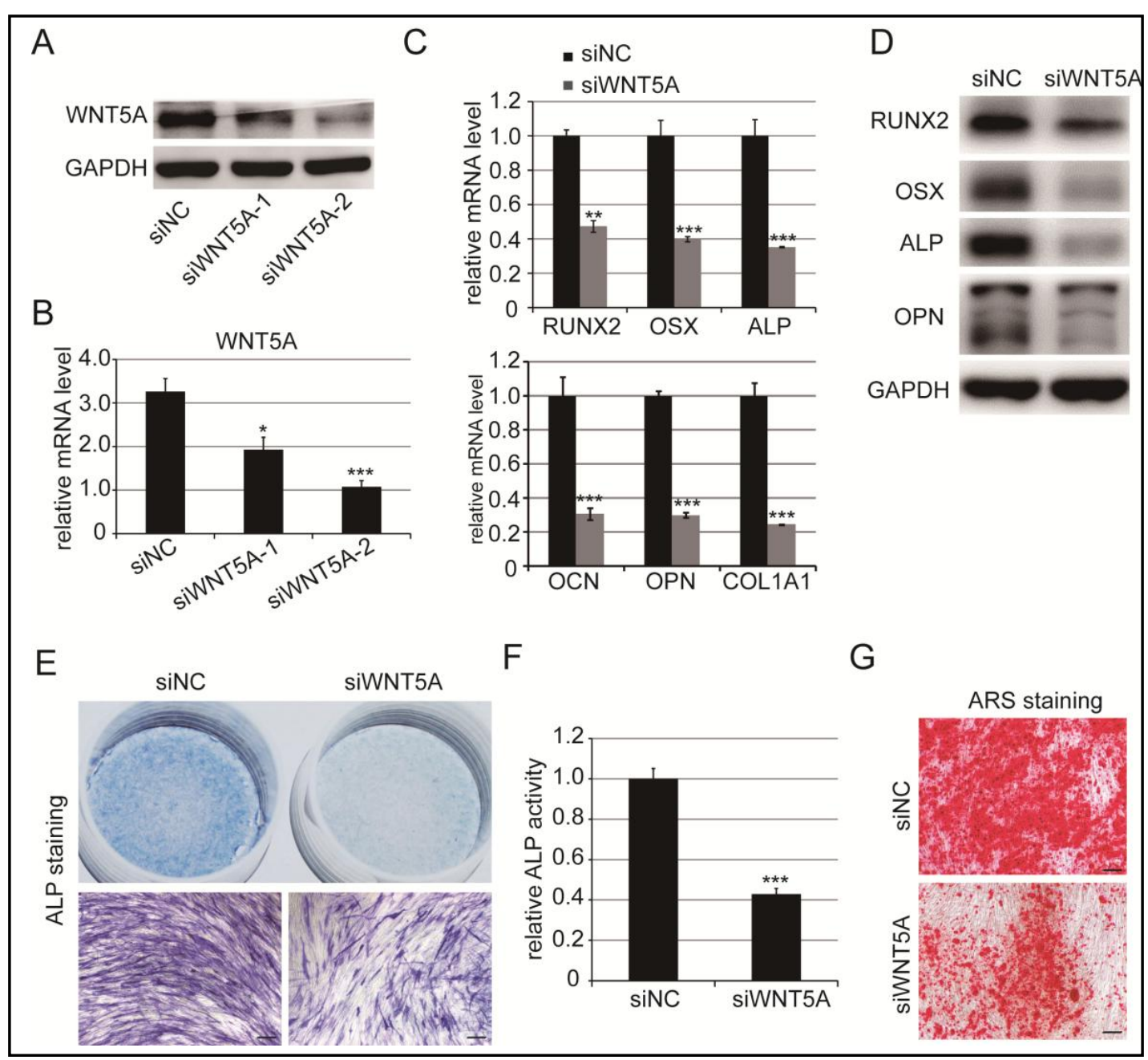

Fig. 5. Downregulation of WNT5A simulated the inhibitory effect of miR-16-2*. (A and B) qRT-PCR and western blotting analyses were used to verify the knockdown efficiency of three siRNAs for WNT5A. (C) qRT-PCR was used to analyze the expression of key transcription factors and osteoblast markers on day 6 of induced cells. (D) Western blotting analysis was used to analyze the protein level of key osteoblast markers. (E and F) ALP staining and ALPase activity assay were performed to indicate ALP expression and ALPase activity on day 6 of induced cells. (G) ARS staining was performed to indicate mineral deposition on day 12 of induced cells. GAPDH was used as an internal control in qRT-PCR and western blot analysis of genes. U6 was used as an internal normalization control of miRNA. Quantitative data were presented as the mean \pm SD $(\mathrm{n}=3),{ }^{*} \mathrm{P}<0.05 ;{ }^{* *} \mathrm{P}<0.01 ;{ }^{* * *} \mathrm{P}<0.001$ compared with their negative control. All the staining and western blot images were confirmed by at least three repeated trails. Scale bar is $100 \mathrm{~mm}$.

showed that DKK1 significantly suppressed the osteogenic differentiation of hBMSCs in a concentration-dependent manner, which was indicated by the reduction of ALP staining, delay mineral deposition, and decrease ALP activity (Fig. 6A, B). Furthermore, the promotion of hBMSCs osteoblast differentiation by miR-16-2* inhibitor was almost abrogates by $1 \mathrm{nM}$ DKK1, as indicated by decreased ALP staining and ALP activity, suppressed the expression of key transcription factors and marker genes of osteoblast differentiation, and blocked mineral deposition (Fig. 6C-E). Then, the activity of the Wnt signaling pathway was detected by TOP/ FOP reporter assay. Results showed that the overexpression of miR-16-2* or interference with endogenous WNT5A decreased the activity of Wnt signaling pathway, while the inhibition of miR-16-2* enhanced it (Fig. 6F). qRT-PCR further confirmed that the expression levels of CTNNB1 and LRP5/ 6 were significantly reduced in the overexpression of miR-16-2*

\section{KARGER}




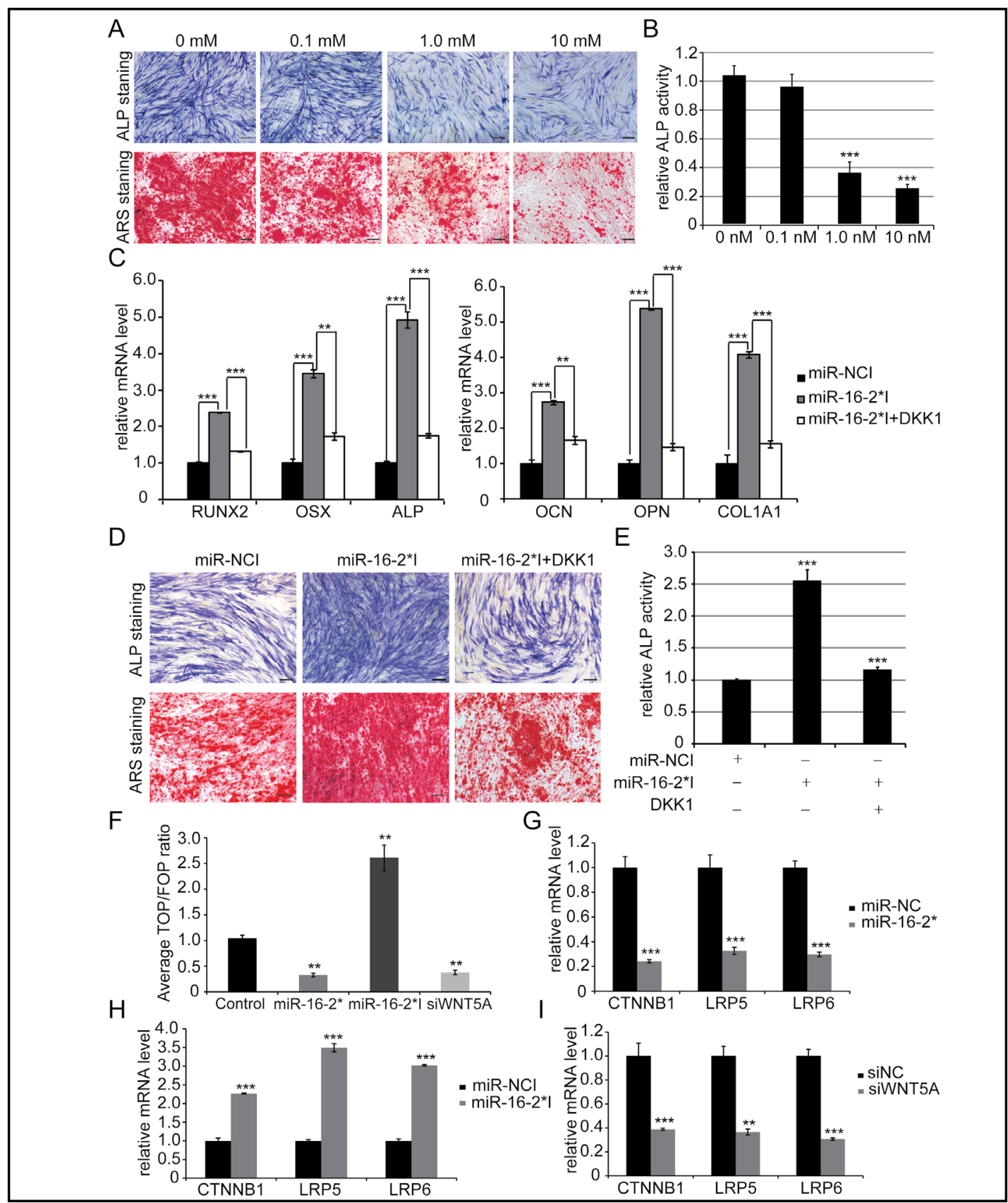

Fig. 6. miR-16-2* interferes with canonical WNT signaling to regulate osteoblast differentiation.(A-B) ALP and ARS staining were performed in induced cells after added different concentrations of DKK1 on day 6 or day 2 of induced cells, respectively. (C) ALP activity assay was performed after added different concentrations of DKK1 on day 6 of induced cells. (D and E) ALP staining and activity were performed in induced cells in which endogenous miR-16-2* was suppressed by miR-16-2* inhibitor and then canonical WNT signaling was block by DKK1. (F) The activity of the Wnt signaling pathway in different treatment groups was detected by TOP/FOP report assay. Cells were transfected with TOPglow and FOPglow expression plasmids. The values of TOPglow and FOPglow were detected and the TOP/FOP ratio reflected the activity of the Wnt signaling pathway. (G-I) qRT-PCR was used to analyze the key genes expression of Wnt signaling pathway. GAPDH was used as an internal control in RT-PCR and western blot analysis of genes. U6 was used as an internal normalization control of miRNA. Quantitative data were presented as the mean \pm SD $(\mathrm{n}=$ 3), ${ }^{*} \mathrm{P}<0.05$; ${ }^{* *} \mathrm{P}<0.01$; ${ }^{* * *} \mathrm{P}<0.001$ compared with their negative control. All the staining and western blot images were confirmed by at least three repeated trails. Scale bar is $100 \mathrm{~mm}$.

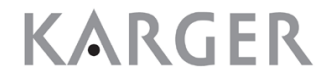




\section{Cellular Physiology Cell Physiol Biochem 2018;51:1087-1102

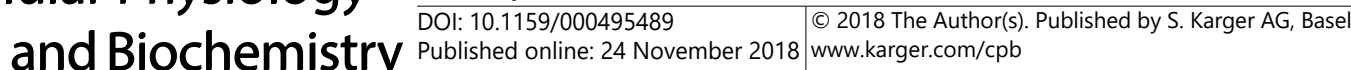 \\ Duan et al.: miR-16-2* Regulates Osteogenesis Via WNT Pathway}

or interference with endogenous WNT5A, and were upregulated in the suppression of miR16-2* (Fig. 6G-I). Summarily, these data further suggest that miR-16-2* interferes with WNT signaling to regulate osteoblast differentiation of hBMSCs.

\section{Discussion}

Despite miR-16-2* has been established as critical factor for diagnosing cervical cancer, the central role of this miRNA in osteogenesis is still far from being elucidated. In this study, we show that mature miR-16-2* levels are abnormally increased in bone of patients with osteoporosis. Forced expression of miRNA-16-2* in mesenchymal stem cells suppressed osteogenesis. miR-16-2* interferes with WNT signaling to regulate osteoblast differentiation though directly suppressing WNT5A. These results are the first to suggest an important role of miR-16-2* in the regulation of MSC osteogenic differentiation, and abnormal expression of miR-16-2* may be responsible for the process of osteogenesis.

Osteoporosis characterized by low bone mineral density and low trauma fractures, is a common and progressive skeletal disorder-related disease in aging people worldwide. By secreting alkaline phosphatase and bone matrix proteins that induce bone matrix mineralization, osteoblasts act as the major cells that contribute to bone formation. Reduce the ability of mesenchymal stem cells (MSCs) differentiate into osteoblast (named as osteogenesis, osteogenic or osteoblast differentiation) would lead to the decrease of bone mineral density and disruption of bone microarchitecture [25], then the imbalance of bone remodeling, and finally result in osteoporosis. Therefore, understanding the underlying mechanism involved in the osteoblast differentiation and activation processes is in urgent need for the treatment of bone formation imbalanced diseases.

Osteogenesis is a complex multi-step process involving the differentiation of MSCs into osteoblast progenitor cells, preosteoblasts, osteoblasts and osteocytes, and the crosstalk between multiple cell types for the formation and remodeling of bone. Differentiation of MSCs into mature osteoblastic cells, regulation of osteoblast functions and the formation of bone by osteoblast cells and their primary functional activities, involve highly regulated processes mediated by a series of multiple signals, including BMPs, TGF $\beta$, PI3K, WNT, NOTCH, as well as other growth factors and hormones [26-29]. BMPs are essential for organogenesis in early and late development. Deletion of BMP ligands [30] or BMP receptors [31] from the limb bud mesenchyme impairs chondrogenic or osteogenic differentiation and induces skeletal patterning defects. A second major molecular pathway involved in osteoblastogenesis is WNT signaling. WNTs are historically categorized by whether they signal in a canonical or non-canonical manner. WNT signaling pathway regulates bone formation through $\beta$-catenindependent canonical and -independent non-canonical signaling pathways. However, complex cross talk between various molecular pathways [32], make it difficult to coordinated regulation osteogenesis differentiation through single gene.

The canonical WNT signaling pathway plays an important role in bone differentiation and bone formation [33-36]. The canonical WNT3A and the non- canonical WNT5A have the effect of promoting bone formation in collaboration with each other. DKK1, an important inhibitor of canonical WNT signaling pathway, also significantly suppressed the osteogenic differentiation of hBMSCs in a concentration-dependent manner. The WNT5A of the noncanonical WNT pathway is also reported to play an important role in bone formation and osteoblast differentiation. WNT5A+/- mice had a reduction in bone mass with a reduced number of osteoblasts, whereas an increased number of adipocytes were seen in the bone marrow. Wnt5a has been shown to induce osteoblastogenesis through the attenuation of PPARG-induced adipogenesis in bone marrow mesenchymal stem cells [37]. WNT5A is critical for the pro-osteogenic effects of iron chelation using deferoxamine [38]. We found that inhibiting WNT5A significantly impaired the ability of MSC to differentiate into osteoblasts. Other studies have found that the canonical WNT3A and the non- canonical WNT5A have the effect of promoting bone formation in collaboration with each other. If 


\section{Cellular Physiology Cell Physiol Biochem 2018;51:1087-1102

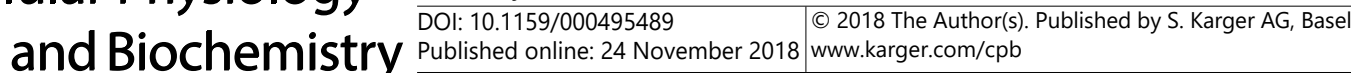 \\ Duan et al.: miR-16-2* Regulates Osteogenesis Via WNT Pathway}

rWNT3A was supplemented during a time window following rWNT5A treatment, osteogenic differentiation was enhanced both in murine and human ESCs [39]. Sphingosine-1-phosphate (S1P) -enhanced Wnt5a promotes low-density lipoprotein receptor-related protein 5 or 6 (LRP5/6) expression, resulting in the trigger of osteogenic differentiation of C3H10T1/2 cells [40]. The lack of Wnt5a in osteoblast-lineage cells impaired Wnt/ $\beta$-catenin signaling due to the reduced expression of LRP5 and LRP6 [41]. These results confirmed a pivotal role of WNT signaling in the regulation of osteoblast differentiation. We demonstrated that downregulate Wnt5a decreased the expression of CTNNB1, LRP5 and LRP6 during osteoblast differentiation of bMSCs.

Recently, miRNAs have been found to be important regulators of osteogenic signaling pathways in multiple aspects and multiple signaling pathways. MiRNAs are short non-coding RNA molecules of 17-25 nucleotides that control gene expression at the post-transcriptional level and have been shown to play critical roles in a wide variety of biological processes, including proliferation, reprogramming, differentiation, cell cycle, invasion and apoptosis $[6,7,9,42-44]$. Each miRNA regulates numerous mRNAs and involve multiple signaling pathways. Increasing evidences have indicated that miRNAs play multiple functions in skeletal development, bone formation and remodeling, and osteoblast differentiation, through regulation of the osteoblastic phenotype related essential signaling pathways [23, 45-48] . For example, under osteogenic differentiation, multiple miRNAs such as miR-17$5 p$ and miR-106a that attenuate RUNX2 and SMAD signal is by suppressing BMP2 [49]. Early expression of miR-195-5p inhibits differentiation and mineralization via altering the gene regulatory network of osteoblast differentiation and impairing the induction of BMP responsive genes. Through targeting BMP-7 and inhibiting BMP-7/PI3K-survivin signaling, miR-542-3p suppresses osteoblast cell proliferation and differentiation, and inhibits bone formation [50]. In osteoblasts, miR-34c targets multiple components of the Notch signaling pathway, including NOTCH1, NOTCH2 and JAG1 in a direct manner, and influences osteoclast differentiation in a non-cell-autonomous fashion [51].

Accumulating evidence suggests that the expression of $\beta$-catenin is, in part, regulated by specific microRNAs (miRNAs). miR-214 was demonstrated to directly interact with the 3'-untranslated region of the $\beta$-catenin gene CTNNB1, and suppressed Wnt/ $\beta$-catenin signaling through the inhibition of $\beta$-catenin [52]. miR-139-5p exerts its role in BMSC osteogenesis most probably through direct targeting CTNNB1 and frizzled 4 (FZD4) [53]. MiR-376c inhibits osteoblastogenesis by targeting WNT3 and ARF-GEF-1 -facilitated augmentation of $\beta$-catenin transactivation [54]. MiR-26a is majorly target on GSK3 $\beta$ to activate WNT signaling for promoting osteogenic differentiation of BMSCs [55]. In this study, we demonstrated that miR-16-2* can suppress osteoblast differentiation of hBMSCs through regulating WNT5A-mediated WNT pathway. MiR-16-2* can be directly targeted to the 3-terminal non-coding region of WNT5A and regulate its expression. Downregulation of WNT5A can simulate the inhibitory effect of miR-16-2* on osteoblast differentiation of hBMSCs. The expression levels of CTNNB1 and LRP5/6 were significantly reduced in the overexpression of miR-16-2* or interference with endogenous WNT5A. The blocking of WNT signal with DKK1 can not only obviously inhibit osteogenesis differentiation, but also partly weaken the promotion of osteogenesis differentiation by suppression the expression of endogenous miR-16-2*. We speculate that WNT5A regulates osteogenesis and may function through both canonical and non-canonical WNT pathways.

\section{Conclusion}

The regulation of osteoblast differentiation is very important for the intervention of osteoporosis. The insafety of gene compilation and the complexity of gene regulation make it difficult to be an effective target for osteoporosis prevention. MiRNA has an advantage in the prevention and treatment of osteoporosis due to its unconformity characteristics, easy synthesis in vitro and multi-target characteristics of regulation. For the first time, we found

\section{KARGER}




\section{Cellular Physiology Cell Physiol Biochem 2018;51:1087-1102 and Biochemistry Published $\begin{aligned} & \text { DOI: } 10.1159 / 000495489 \\ & \text { (c) } 2018 \text { The Author(s). Published by S. Karger AG, Basel } \\ & \text { www.karger.com/cpb }\end{aligned}$

that miR-16-2* was closely related to osteoporosis and played a pivotal role in osteoblast differentiation. miR-16-2* are abnormally increased in bone of patients with osteoporosis, and can significant impair the ability of MSC to differentiate into osteoblasts, by direct targeting WNT5A and subsequently blocking canonical and non-canonical WNT pathways. Our findings may provide a promising therapeutic target against disorders associated with bone formation.

\section{Acknowledgements}

LJ and XY participated in the design of the study; LJ, HZ, YX, XT, YY, ZH and CL carried out the experiments; LJ and SC analyzed the data and performed the statistical analysis; LJ and XY drafted the manuscript. All authors read and approved the final manuscript.

\section{Disclosure Statement}

The authors declare no conflicts of interest.

\section{References}

1 Feng Z, Liu C, Guan X, Mor V: China's rapidly aging population creates policy challenges in shaping a viable long-term care system. Health Aff (Millwood) 2012;31:2764-2773.

-2 Raisz LG: Pathogenesis of osteoporosis: Concepts, conflicts, and prospects. J Clin Invest 2005;115:33183325.

-3 Rodan GA, Martin TJ: Therapeutic approaches to bone diseases. Science 2000;289:1508-1514.

4 Small EM, Olson EN: Pervasive roles of micrornas in cardiovascular biology. Nature 2011;469:336-342.

-5 Heinrich EM, Dimmeler S: Micrornas and stem cells: Control of pluripotency, reprogramming, and lineage commitment. Circ Res 2012;110:1014-1022.

-6 Hyun J, Wang S, Kim J, Rao KM, Park SY, Chung I, Ha CS, Kim SW, Yun YH, Jung Y: Microrna-378 limits activation of hepatic stellate cells and liver fibrosis by suppressing gli3 expression. Nat Commun 2016;7:10993.

7 Cui Y, Han J, Xiao Z, Chen T, Wang B, Chen B, Liu S, Han S, Fang Y, Wei J, Wang X, Ma X, Dai J: The mir-20-restwnt signaling axis regulates neural progenitor cell differentiation. Sci Rep 2016;6:23300.

8 Gao Y, Chen L, Song H, Chen Y, Wang R, Feng B: A double-negative feedback loop between e2f3b and mir- 200b regulates docetaxel chemosensitivity of human lung adenocarcinoma cells. Oncotarget 2016;7:27613-27626.

9 Ma L, Ma S, Zhao G, Yang L, Zhang P, Yi Q, Cheng S: Mir-708/lsd1 axis regulates the proliferation and invasion of breast cancer cells. Cancer Med 2016;5:684-692.

10 van Rooij E: The art of microrna research. Circ Res 2011;108:219-234.

$>11$ Chen J, Qiu M, Dou C, Cao Z, Dong S: Micrornas in bone balance and osteoporosis. Drug Dev Res 2015;76:235-245.

12 Fang S, Deng Y, Gu P, Fan X: Micrornas regulate bone development and regeneration. Int J Mol Sci 2015;16:8227-8253.

13 Xie Y, Zhang L, Gao Y, Ge W, Tang P: The multiple roles of microrna-223 in regulating bone metabolism. Molecules 2015;20:19433-19448.

14 Hassan MQ Tye CE, Stein GS, Lian JB: Non-coding rnas: Epigenetic regulators of bone development and homeostasis. Bone 2015;81:746-756.

15 Calin GA, Ferracin M, Cimmino A, Di Leva G, Shimizu M, Wojcik SE, Iorio MV, Visone R, Sever NI, Fabbri M, Iuliano R, Palumbo T, Pichiorri F, Roldo C, Garzon R, Sevignani C, Rassenti L, Alder H, Volinia S, Liu CG, Kipps TJ, Negrini M, Croce CM: A microrna signature associated with prognosis and progression in chronic lymphocytic leukemia. N Engl J Med 2005;353:1793-1801. 


\section{Cellular Physiology Cell Physiol Biochem 2018;51:1087-1102

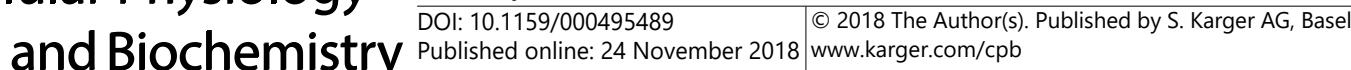

16 Cimmino A, Calin GA, Fabbri M, Iorio MV, Ferracin M, Shimizu M, Wojcik SE, Aqeilan RI, Zupo S, Dono M, Rassenti L, Alder H, Volinia S, Liu CG, Kipps TJ, Negrini M, Croce CM: Mir-15 and mir-16 induce apoptosis by targeting bcl2 Proc Natl Acad Sci U S A 2005;102:13944-13949.

17 Aqeilan RI, Calin GA, Croce CM: Mir-15a and mir-16-1 in cancer: Discovery, function and future perspectives. Cell Death Differ 2010;17:215-220.

18 Calin GA, Dumitru CD, Shimizu M, Bichi R, Zupo S, Noch E, Aldler H, Rattan S, Keating M, Rai K, Rassenti L, Kipps T, Negrini M, Bullrich F, Croce CM: Frequent deletions and down-regulation of micro- rna genes mir15 and mir16 at 13q14 in chronic lymphocytic leukemia. Proc Natl Acad Sci U S A 2002;99:1552415529.

-19 El-Tawdi AH, Matboli M, Shehata HH, Tash F, El-Khazragy N, Azazy Ael S, Abdel-Rahman O: Evaluation of circulatory rna-based biomarker panel in hepatocellular carcinoma. Mol Diagn Ther 2016;20:265-277.

20 Lovat F, Fassan M, Gasparini P, Rizzotto L, Cascione L, Pizzi M, Vicentini C, Balatti V, Palmieri D, Costinean S, Croce CM: Mir-15b/16-2 deletion promotes b-cell malignancies. Proc Natl Acad Sci U S A 2015;112:1163611641.

- 21 Zhang Y, Zhang D, Wang F, Xu D, Guo Y, Cui W: Serum mirnas panel (mir-16-2*, mir-195, mir-2861, mir-497) as novel non-invasive biomarkers for detection of cervical cancer. Sci Rep 2015;5:17942.

-22 Li D, Liu J, Guo B, Liang C, Dang L, Lu C, He X, Cheung HY, Xu L, He B, Liu B, Shaikh AB, Li F, Wang L, Yang Z, Au DW, Peng S, Zhang Z, Zhang BT, Pan X et al.: Osteoclast-derived exosomal mir-214-3p inhibits osteoblastic bone formation. Nat Commun 2016;7:10872.

-23 Li H, Li T, Fan J, Fan L, Wang S, Weng X, Han Q Zhao RC: Mir-216a rescues dexamethasone suppression of osteogenesis, promotes osteoblast differentiation and enhances bone formation, by regulating c-cblmediated pi3k/akt pathway. Cell Death Differ 2015;22:1935-1945.

24 Gregory CA, Gunn WG, Peister A, Prockop DJ: An alizarin red-based assay of mineralization by adherent cells in culture: Comparison with cetylpyridinium chloride extraction. Anal Biochem 2004;329:77-84.

25 Boyle WJ, Simonet WS, Lacey DL: Osteoclast differentiation and activation. Nature 2003;423:337-342.

-26 Lin GL, Hankenson KD: Integration of bmp, wnt, and notch signaling pathways in osteoblast differentiation. J Cell Biochem 2011;112:3491-3501.

27 Hu H, Hilton MJ, Tu X, Yu K, Ornitz DM, Long F: Sequential roles of hedgehog and wnt signaling in osteoblast development. Development 2005;132:49-60.

28 Guntur AR, Rosen CJ: The skeleton: A multi-functional complex organ: New insights into osteoblasts and their role in bone formation: The central role of pi3kinase. J Endocrinol 2011;211:123-130.

29 Chen G, Deng C, Li YP: Tgf-beta and bmp signaling in osteoblast differentiation and bone formation. Int J Biol Sci 2012;8:272-288.

-30 Bandyopadhyay A, Tsuji K, Cox K, Harfe BD, Rosen V, Tabin CJ: Genetic analysis of the roles of bmp2, bmp4, and bmp7 in limb patterning and skeletogenesis. PLoS Genet 2006;2:e216.

-31 Yoon BS, Ovchinnikov DA, Yoshii I, Mishina Y, Behringer RR, Lyons KM: Bmpr1a and bmpr1b have overlapping functions and are essential for chondrogenesis in vivo. Proc Natl Acad Sci U S A 2005;102:5062-5067.

-32 Ekstrom JE, Shuman WP, Mack LA: Mr imaging of accessory soleus muscle. J Comput Assist Tomogr 1990;14:239-242.

33 Davis LA, Zur Nieden NI: Mesodermal fate decisions of a stem cell: The wnt switch. Cell Mol Life Sci 2008;65:2658-2674.

-34 Cui Y, Niziolek PJ, MacDonald BT, Zylstra CR, Alenina N, Robinson DR, Zhong Z, Matthes S, Jacobsen CM, Conlon RA, Brommage R, Liu Q, Mseeh F, Powell DR, Yang QM, Zambrowicz B, Gerrits H, Gossen JA, He X, Bader M, Williams BO, Warman ML, Robling AG: Lrp5 functions in bone to regulate bone mass. Nat Med 2011;17:684-691.

35 Qiu W, Andersen TE, Bollerslev J, Mandrup S, Abdallah BM, Kassem M: Patients with high bone mass phenotype exhibit enhanced osteoblast differentiation and inhibition of adipogenesis of human mesenchymal stem cells. J Bone Miner Res 2007;22:1720-1731.

-36 Westendorf JJ, Kahler RA, Schroeder TM: Wnt signaling in osteoblasts and bone diseases. Gene 2004;341:19-39. 


\section{Cellular Physiology Cell Physiol Biochem 2018;51:1087-1102

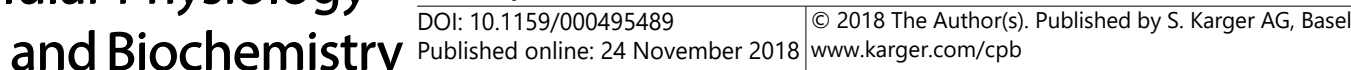

-37 Takada I, Mihara M, Suzawa M, Ohtake F, Kobayashi S, Igarashi M, Youn MY, Takeyama K, Nakamura T, Mezaki Y, Takezawa S, Yogiashi Y, Kitagawa H, Yamada G, Takada S, Minami Y, Shibuya H, Matsumoto K, Kato S: A histone lysine methyltransferase activated by non-canonical wnt signalling suppresses ppar-gamma transactivation. Nat Cell Biol 2007;9:1273-1285.

-38 Baschant U, Rauner M, Balaian E, Weidner H, Roetto A, Platzbecker U, Hofbauer LC: Wnt5a is a key target for the pro-osteogenic effects of iron chelation on osteoblast progenitors. Haematologica 2016;101:14991507.

-39 Keller KC, Ding H, Tieu R, Sparks NR, Ehnes DD, Zur Nieden NI: Wnt5a supports osteogenic lineage decisions in embryonic stem cells. Stem Cells Dev 2016;25:1020-1032.

-40 Hashimoto Y, Kobayashi M, Matsuzaki E, Higashi K, Takahashi-Yanaga F, Takano A, Hirata M, Nishimura F: Sphingosine-1-phosphate-enhanced wnt5a promotes osteogenic differentiation in c3h10t1/2 cells. Cell Biol Int 2016;40:1129-1136.

41 Okamoto M, Udagawa N, Uehara S, Maeda K, Yamashita T, Nakamichi Y, Kato H, Saito N, Minami Y, Takahashi N, Kobayashi Y: Noncanonical wnt5a enhances wnt/beta-catenin signaling during osteoblastogenesis. Sci Rep 2014;4:4493.

-42 Zhang BL, Dong FL, Guo TW, Gu XH, Huang LY, Gao DS: Mirnas mediate gdnf-induced proliferation and migration of glioma cells. Cell Physiol Biochem 2017;44:1923-1938.

43 Xiao C, Calado DP, Galler G, Thai TH, Patterson HC, Wang J, Rajewsky N, Bender TP, Rajewsky K: Mir-150 controls b cell differentiation by targeting the transcription factor c-myb. Cell 2007;131:146-159.

44 Parchem RJ, Ye J, Judson RL, LaRussa MF, Krishnakumar R, Blelloch A, Oldham MC, Blelloch R: Two mirna clusters reveal alternative paths in late-stage reprogramming. Cell Stem Cell 2014;14:617-631.

45 Chang H, Wang Y, Liu H, Nan X, Wong S, Peng S, Gu Y, Zhao H, Feng H: Mutant runx2 regulates amelogenesis and osteogenesis through a mir-185-5p-dlx2 axis. Cell Death Dis 2017;8:3221.

46 Sun YX, Zhang JF, Xu J, Xu LL, Wu TY, Wang B, Pan XH, Li G: Microrna-144-3p inhibits bone formation in distraction osteogenesis through targeting connexin 43. Oncotarget 2017;8:89913-89922.

-47 Zhang L, Tang Y, Zhu X, Tu T, Sui L, Han Q Yu L, Meng S, Zheng L, Valverde P, Tang J, Murray D, Zhou X, Drissi $\mathrm{H}$, Dard MM, Tu Q Chen J: Overexpression of mir-335-5p promotes bone formation and regeneration in mice. J Bone Miner Res 2017;32:2466-2475.

48 Wang X, Guo B, Li Q, Peng J, Yang Z, Wang A, Li D, Hou Z, Lv K, Kan G, Cao H, Wu H, Song J, Pan X, Sun Q, Ling S, Li Y, Zhu M, Zhang P, Peng S et al.: Mir-214 targets atf4 to inhibit bone formation. Nat Med 2013;19:93100.

49 Li H, Li T, Wang S, Wei J, Fan J, Li J, Han Q Liao L, Shao C, Zhao RC: Mir-17-5p and mir-106a are involved in the balance between osteogenic and adipogenic differentiation of adipose-derived mesenchymal stem cells. Stem Cell Res 2013;10:313-324.

50 Kureel J, Dixit M, Tyagi AM, Mansoori MN, Srivastava K, Raghuvanshi A, Maurya R, Trivedi R, Goel A, Singh D: Mir-542-3p suppresses osteoblast cell proliferation and differentiation, targets bmp-7 signaling and inhibits bone formation. Cell Death Dis 2014;5:e1050.

51 Bae Y, Yang T, Zeng HC, Campeau PM, Chen Y, Bertin T, Dawson BC, Munivez E, Tao J, Lee BH: Mirna-34c regulates notch signaling during bone development. Hum Mol Genet 2012;21:2991-3000.

-52 Cao F, Zhan J, Chen X, Zhang K, Lai R, Feng Z: Mir-214 promotes periodontal ligament stem cell osteoblastic differentiation by modulating wnt/betacatenin signaling. Mol Med Rep 2017;16:9301-9308.

53 Long H, Sun B, Cheng L, Zhao S, Zhu Y, Zhao R, Zhu J: Mir-139-5p represses bmsc osteogenesis via targeting wnt/beta-catenin signaling pathway. DNA Cell Biol 2017;36:715-724.

54 Kureel J, John AA, Prakash R, Singh D: Mir 376c inhibits osteoblastogenesis by targeting wnt3 and arf-gef-1 -facilitated augmentation of beta-catenin transactivation. J Cell Biochem 2018;119:3293-3303.

55 Su X, Liao L, Shuai Y, Jing H, Liu S, Zhou H, Liu Y, Jin Y: Mir-26a functions oppositely in osteogenic differentiation of bmscs and adscs depending on distinct activation and roles of wht and bmp signaling pathway. Cell Death Dis 2015;6:e1851. 